\title{
Measurement loop, automatic tuning and product quality tracking
}

\author{
Marcela Man Dietrich ${ }^{1, *}$ and Marcel Sabin Popa $^{2}$ \\ ${ }^{1}$ Helix GmbH, Hanauer Landstraße 52, 60314 Frankfurt am Main, Germany \\ ${ }^{2}$ Faculty of Machine Building, Technical University of Cluj-Napoca, B-dul Muncii nr. 103-105, \\ 400641 Cluj-Napoca, Romania
}

\begin{abstract}
Starting from the PDCA cycle (Plan - Do - Check - Act), imposed by the ISO 9000 quality standards, a loop is proposed to automatically adjust the work equipment to the statistical distribution of the quality feature of the last $\mathrm{N}$ products randomly selected. The loop promptly warns the leadership in case of distortion of the statistical distribution, caused by equipment, raw materials, human operators or the environment. It also constantly informs about the percentage of noncompliant products. The main original element, at the heart of the article's concerns, is the quality tracking loop presented in section 4 . The starting point is the quality management scheme, according to ISO9000, while sections 2-3 expose the theoretical basis of the subject, without which it would have not been possible to deepen the role of the modules from the loop proposed in Figure 6.
\end{abstract}

\section{Introduction}

The current standard, concerning quality management systems, maintains the requirements of the old standards, but groups them logically on the technological flow.

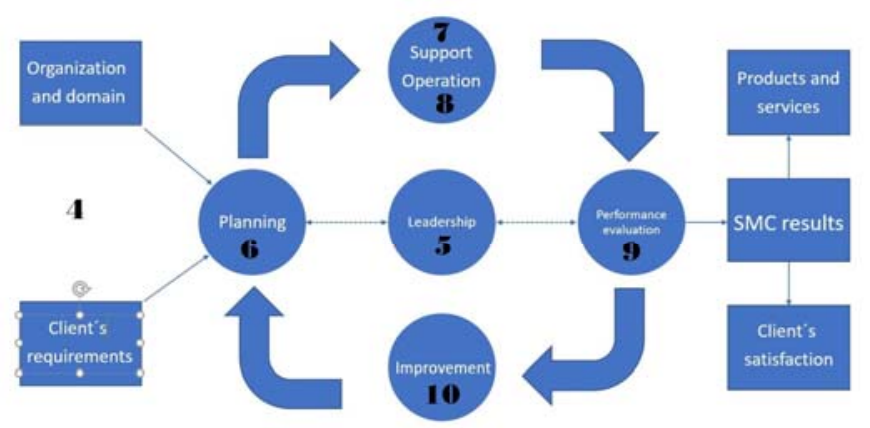

Fig. 1. Quality management in the PDCA cycle, [1].

\footnotetext{
${ }^{*}$ Corresponding author: marcela man@hotmail.com
} 
The process-based approach incorporates the PDCA cycle, the requirements being grouped into 7 articles, corresponding to numbers 4 to 10 in Figure 1. The cycle Plan- DoCheck- Act is a typical automation loop, in which the Check- Act operations are a feedback that interferes with planning, in order to improve the quality of the product.

Figure 2 only aims to redraw Figure 1, highlighting that the PDCA cycle is a typical automation loop.

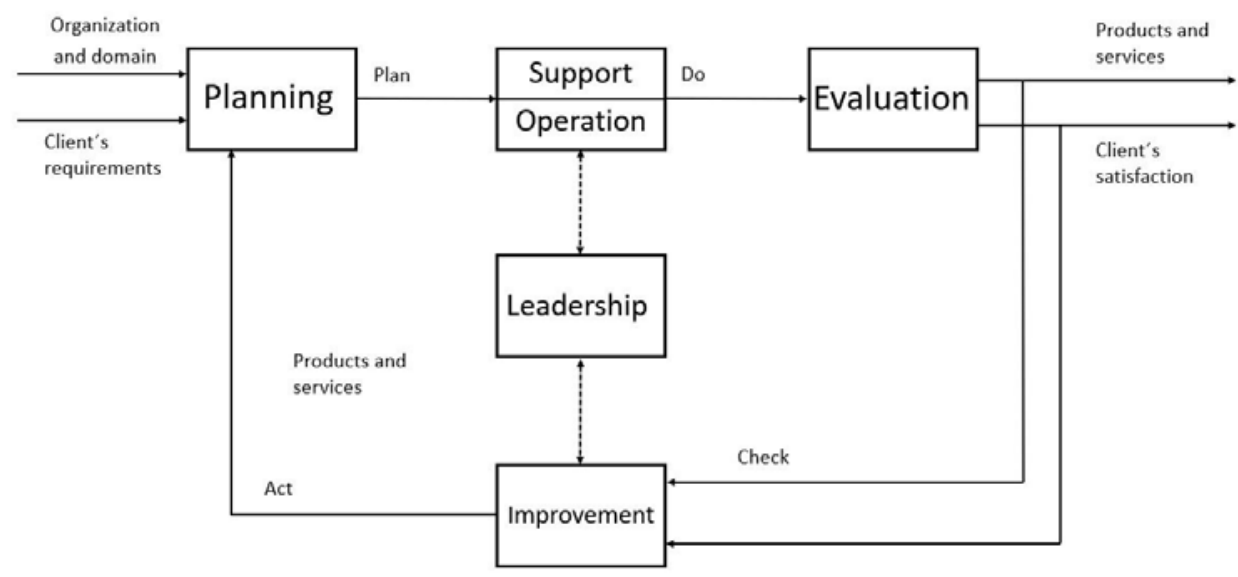

Fig. 2. PDCA cycle as an automation loop.

Since the standard [1] has a general nature, covering all activities in production and services, this paper aims to identify solutions for the "Improvement" module, so that through "Verification" and "Actuation", some operations from the quality management system of a production line can be automated.

\section{Statistical distributions}

In Figure 2, the verification consists of measuring the quality of the product, depending on which action will be taken for improvement. Inevitably, factors (equipment, raw materials, human operator or environment) interfere with the process, which, through their variation, make the products of variable quality characteristics.

Acting after checking a single product would be a mistake, leading to instability in the line performance. The goal of obtaining identical products with the model is realistically replaced with the probability that the product will have the (measurable) quality characteristic, defined as $\mathrm{X} \in\left[\mathrm{X}_{\mathrm{m}}, \mathrm{X}_{\mathrm{M}}\right]$, in a pre-set domain: $X \epsilon\left[X_{i}, X_{s}\right][6]$.

In which case it will be assessed that the product is in conformity (or, also called compliance).

The probability density $f(x ; \theta) \geq 0$, where $\Theta$ is a parameter that individualizes the distribution, has a variation as in Figure 3.

The compliant product is obtained with a probability equal to the $A_{2}$ area (1):

$$
p=A_{2}=\int_{X i}^{X s} f(x ; \theta) d x
$$




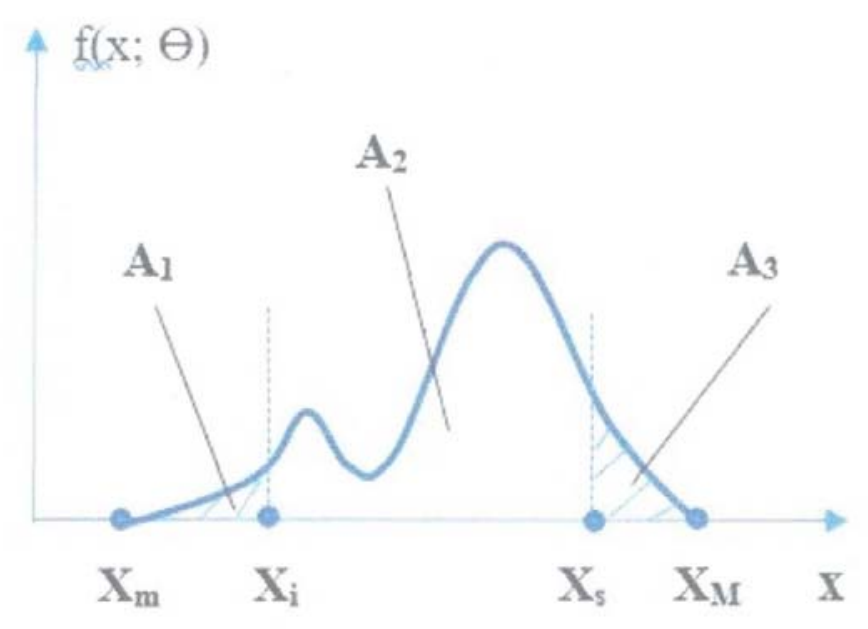

Fig. 3. Continuous statistical distribution.

The probability that the product has the characteristic in its definition domain is a certainty (2):

$$
A_{1}+A_{2}+A_{3}=\int_{X m}{ }^{X M} f(x ; \theta) d x=1
$$

So, the probability of obtaining non-compliant (non-conformable) products is (3):

$$
q=A_{1}+A_{3}=\int_{X m}{ }_{i} f(x ; \theta) d x+\int_{X s}{ }^{X} f(x ; \theta) d x=1-p
$$

\section{Normal Gauss distribution}

The probability density is not completely defined by one $\Theta$ parameter, but by two parameters: [5]

- $\quad$ arithmetic mean resulting from " $n$ " measurements (4):

$$
\mu=\left(\sum_{i=1}^{n} x_{i}\right) / n
$$

- $\quad$ dispersion (standard deviation) (5):

$$
\sigma=\sqrt{ }\left\{\left[\sum_{i=1}^{n}\left(x_{i}-\mu\right)^{2}\right] / n\right\}
$$

The probability density, at normal distribution, is (6):

$$
f(x ; \mu ; \sigma)=\left[1 /(\sigma \sqrt{ }(2 \pi)] e^{-1 / 2[(x-\mu) / \sigma][(x-\mu) / \sigma]}\right.
$$

And the curve of Figure 4 is symmetrical to the $x=\mu$ axis (7):

$$
f(\mu-\alpha ; \mu, \sigma)=f(\mu+\alpha ; \mu, \sigma)
$$




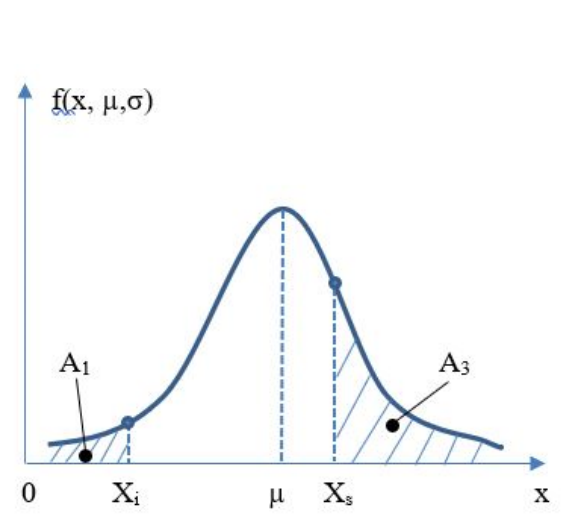

Fig. 4. Gauss probability density.

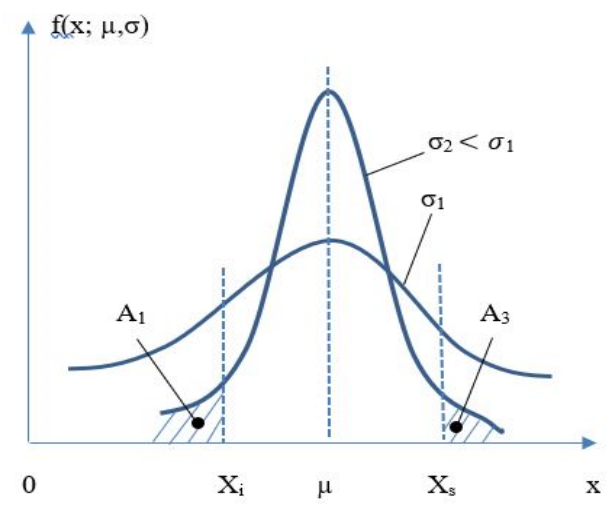

Fig. 5. The influence of dispersion on probability density.

The Gauss probability density curve has a maximum at $\mathrm{x}=\mu$. At the same average value $\mu$, the curve with the $\sigma_{2}<\sigma_{1}$ dispersion is sharper (Figure 5).

Both curves have the areas below the graph equal to the unit, so at a lower dispersion the $\mathrm{A}_{1}+\mathrm{A}_{3}$ probability of nonconformities decreases.

A distribution like the one in Figure 3 is not normal. The degree of deviation from the Gauss distribution is given by the $\chi^{2}$ calc test, defined as [3] (8):

$$
\chi_{\text {calc }}^{2}=\sum_{j=1}^{n}\left[\left(N_{j}-N_{j 0}\right)^{2} / N_{j 0}\right]
$$

Where $\mathrm{N}_{\mathrm{j}}$ - the number of measurements in the $\mathrm{j}$ class range;

$\mathrm{N}_{\mathrm{j} 0}$ - the number of measurements in the same interval, if the distribution was normal.

Thus, if $\sigma$ expresses the measurement deviations from the mean value $\mu, \chi^{2}$ calc expresses the deviation from the values of the Gauss curve and gives an objective indication of the degree of distribution distortion.

\section{Automation of quality management}

The scheme from Figure 6 proposes a measurement loop, auto-tuning and quality tracking that includes several modules.

Random measurement program. Initiates the measurement of the quality characteristic of a product, either by time, or by counting the products [4].

The measuring equipment picks up the random piece and measures its quality feature. The precision class of the equipment must be superior to the precision class of the equipment embedded in the production line command.

The register is initialized with a number of measurements (9):

$$
X_{I}=\ldots=X_{N}=\left(X_{i}+X_{S}\right) / 2
$$

Large enough to count on a statistical distribution. The $\mathrm{X}_{\mathrm{N}+1}$ measurement will replace the oldest value, $\mathrm{X}_{1}$, so the register will store the last $\mathrm{N}$ measurements.

The database stores Gauss distributions $\mathrm{f}(\mathrm{x}, \mu, \sigma)$ and tabulated $\chi_{\text {tab }}^{2}$ values, up to which the distribution can be considered normal [3].

The computer recalculates, after each new measurement, the values $\mu, \sigma$ and $\chi^{2}$ calc, with the relations (4), (5) and (8). 
The stabilizer module checks if $\chi^{2}$ calc $<\chi_{\text {tab }}^{2}$ If the relation is not met, the distribution is distorted (Figure 3).

At least one of the disturbing factors of the process has sudden and significant variations, so that the product quality characteristic is not in a predictable and acceptable range. For example:

- the equipment includes two differently adjusted machines;

- two process-measuring and control equipment have different calibrations or one is not properly matched;

- raw materials come from different lots or are uneven;

- the intervening human operators use different working methods, or one is not properly trained;

- the environment has its temperature, humidity, etc. likely to significantly influence the quality characteristic and these sizes are not controlled [2].

The leadership is alerted and technology engineers will identify and eliminate distortions (Figure 4).

The centring module corrects the initial $\left(\mathrm{X}_{\mathrm{i}}+\mathrm{X}_{\mathrm{s}}\right) / 2$ setting off the work equipment with the $\mu-\left[\left(\mathrm{X}_{\mathrm{i}}+\mathrm{X}_{\mathrm{s}}\right) / 2\right]$ value. By bringing the mean value $\mu$ into the middle of the compliance interval, the Gauss curve with symmetry with the $\mathrm{x}=\mathrm{y}$ axis, the percentage of nonconformities is automatically reduced to a minimum (Figure 5).

The optimization module receives from the computer the $\mathrm{q}_{0}(\sigma)$ value of the product non-conformity probability, after centring. Reducing this probability is done by lowering the dispersion $\sigma$ (Figure 5), the decision being taken by the leadership, following an analysis of the involved costs (investments plus losses due to poor product quality).

Example of investments:

- more advanced work equipment;

- measuring device with a superior precision class;

- quality raw materials with high homogeneity;

- trained and motivated human operators;

- controlled environment.

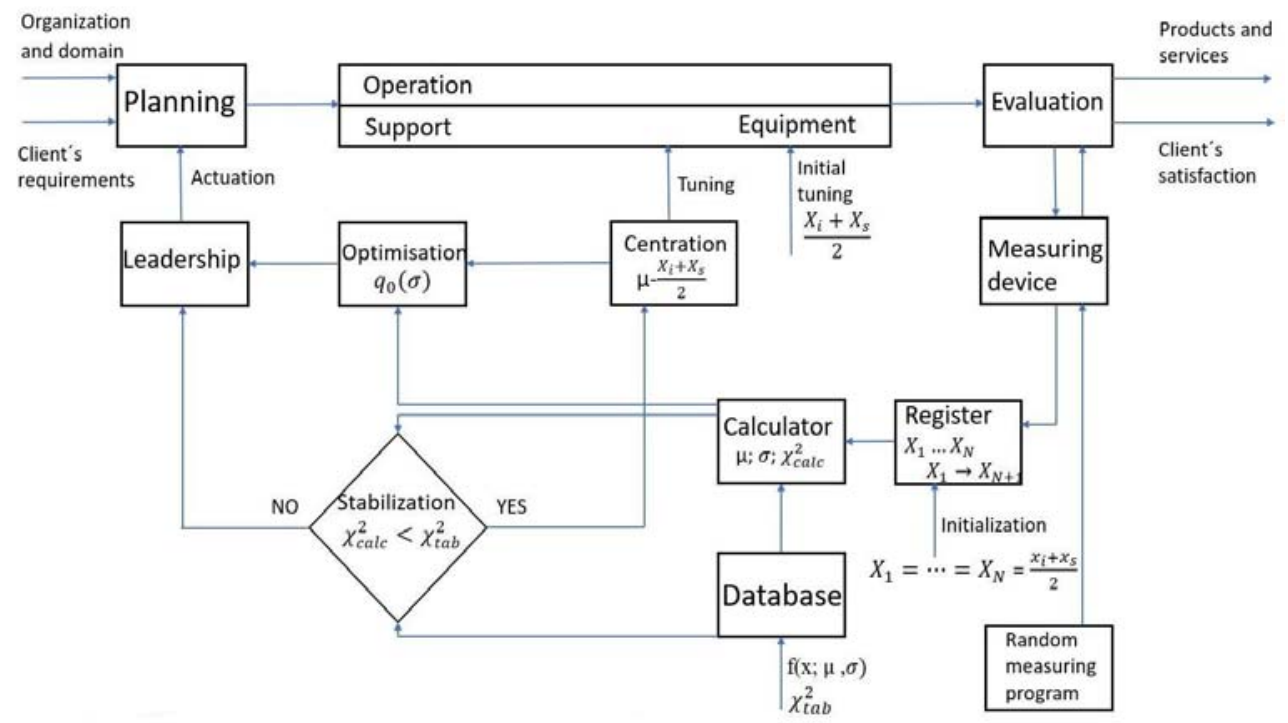

Fig. 6. Measuring loop, automatic tuning and quality tracking. 


\section{Conclusions}

The automation loop proposed for quality management promptly warns the leadership of the appearance of perturbations that distort the distribution of the quality feature.

It is automatically intervened to adjust the work equipment, in order to centre the distribution, based on the last $\mathrm{N}$ measurements and their statistical distribution.

The leadership is foremost aware of the $\mathrm{q}_{0}(\sigma)$ probability of product nonconformity to keep the percentage of nonconformities below planned levels. As a main step to develop my research in the future, the work could continue by designing logical schemes and programs that convey the measurement loop, automatic tuning and quality tracking blocks.

\section{References}

1. *** Quality management systems - fundamental and vocabulary principles(in Romanian) (SR EN ISO 9000: 2015)

2. M. Man Dietrich, M.S. Popa, Current state in the research on electronic monitoring systems for the security and flow of objects and individuals, MATEC Web of Conferences 112, 09006 (2017)

3. M. Buracu, V.Gh. Voda, Modern techniques in statistical quality control (in Romanian) (Editura Tehnică, Bucuresti, 1989)

4. S. Vianello, Controllo di qualita e statistica nell'industria farmaceutica. Tecniche nuove (Milano, 2003)

5. K. Stout, Quality Control in Automation (Kogan Page, London, 1985)

6. M.S. Popa, A. Kunz, T. Kennel, G. Contiu, Tehnologii inovative şi Procese Creative de Productie - Innovative Technologien und Kreative Produktionsprozesse (bilingual) (U.T. Press, Cluj-Napoca, 2009) 